\title{
REVERSES HARDY-TYPE INEQUALITIES VIA JENSEN INTEGRAL INEQUALITY
}

\author{
BOUHARKET BENAISSA ${ }^{1,2^{*}}$, AISSA BENGUESSOUM ${ }^{3,4}$ \\ ${ }^{1}$ Faculty of Material Sciences, University of Tiaret-Algeria. \\ ${ }^{2}$ Laboratory of Informatics and Mathematics, University of Tiaret. Algeria \\ ${ }^{3}$ Faculty of Technologic Sciences, University of Tiaret-Algeria. \\ ${ }^{4}$ Laboratory of Analysis and Control of PDE, University of Sidi Bel-Abbes. Algeria \\ *Corresponding author-mail: Bouharket.benaissa@univ-tiaret.dz
}

\section{DOI: 10.20948/mathmontis-2021-52-5}

Summary. The integral inequalities concerning the inverse Hardy inequalities have been studied by a large number of authors during this century, of these articles have appeared, the work of Sulaiman in 2012, followed by Banyat Sroysang who gave an extension to these inequalities in 2013. In 2020 B. Benaissa presented a generalization of inverse Hardy inequalities. In this article, we establish a new generalization of these inequalities by introducing a weight function and a second parameter. The results will be proved using the Hölder inequality and the Jensen integral inequality. Several the reverses weighted Hardy's type inequalities and the reverses Hardy's type inequalities were derived from the main results.

\section{INTRODUCTION}

In recent years, several researchers have obtained extensions and generalizations of Hardy's inequality in the literature, for more details see [1], Hardy type inequalities for fractional integral operators [2], Hardy type inequalities involving functions of two variables [3]- [4], Hardy-type inequalities via the Steklov operator [5], a new version of inverse Hardy inequalities on time scales that appeared in 2021 see [6]- [7] . Many researchers have obtained results of refinements and generalizations of inverse Hardy inequalities, in 2020, B. Benaissa presented the following generalizations [8, Theorem 2.2].

Let $f, g$ be positive functions defined on $[\mathrm{a} ; \mathrm{b}]$ and $\mathrm{F}(x)=\int_{a}^{x} f(t) d t$. If $\mathrm{g}$ is nondecreasing, then for $\mathrm{p} \geq 1$,

$$
p \int_{a}^{b} \frac{F(x)^{p}}{g(x)} d x \leq(b-a)^{p} \int_{a}^{b} \frac{f(x)^{p}}{g(x)} d x-\int_{a}^{b} \frac{(x-a)^{p}}{g(x)} f(x)^{p} d x,
$$

for $0<p<1$,

$$
p \int_{a}^{b} \frac{F(x)^{p}}{g(x)} d x \geq \frac{(b-a)^{p}}{g(b)} \int_{a}^{b} f(x)^{p} d x-\frac{1}{g(b)} \int_{a}^{b}(x-a)^{p} f(x)^{p} d x
$$

Taking $\mathrm{g}(\mathrm{x})=x^{p}$, we get Sulaiman result inequalities, [9, theorem 3.1] and if we putting $\mathrm{g}(\mathrm{x})=x^{q} ; \mathrm{q}>0$, we get Banyat Sroysang result inequalities, [10, theorem 2.1 and theorem 2.2]. On the other hand, convex functions play an important role in inequality theory, this class of functions has many applications in different mathematical branches (numerical 
calculation, probability theory,...), match results are obtained by the Jensen inequality and many articles relating to different versions of this inequality have been published see for example [11]. Motivated by above literature, in this work we give a generalization of Hardy's integral inequality by using a weight function $\mu$ and a second parameter $q$, this results will be proved by Hölder inequality and Jensen integral inequality.

All along this paper, $f, g$ are measurable non-negatives functions on interval $(a ; b)$ where $0<a<b<+\infty$ and $\mu$ is a weight function (measurable and positive) on $(a ; b)$. In the set of monotone functions, non-increasing (non-decreasing) function means the function is decreasing (increasing) or constant.

\section{PRELIMINARIES}

In this section we state the following Lemmas which are useful in the proofs of main Theorems.

Lemma2.1. Let $0<p \leq q<\infty$ and $f$, $g$, wbe non-negative measurable functions on $(a, b)$ and suppose that $0<\int_{\boldsymbol{a}}^{\boldsymbol{b}} f^{q}(t) \mu(t) d t<\infty$, then

$$
\int_{a}^{b} f^{p}(t) \mu(t) d t \leq\left(\int_{a}^{b} \mu(t) d t\right)^{\frac{q-p}{q}}\left(\int_{a}^{b} f^{p}(t) \mu(t) d t\right)^{\frac{p}{q}} .
$$

The inequality (3) holds for $-\infty<q \leq p<0$ and inverted for $0<q \leq p<\infty$.

Proof. Using Hölder inequality for using the parameter $\frac{q}{p} \geq 1$, we have

$$
\begin{gathered}
\int_{a}^{b} f^{p}(t) \mu(t) d t=\left(\int_{a}^{b} \mu^{\frac{q-p}{q}}(t) d t\right)\left(\int_{a}^{b} f^{p}(t) \mu^{\frac{p}{q}}(t) d t\right) . \\
\leq\left(\int_{a}^{b} \mu(t) d t\right)^{\frac{q-p}{q}}\left(\int_{a}^{b} f^{p}(t) \mu(t) d t\right)^{\frac{p}{q}} .
\end{gathered}
$$

(See the version on time scales in [6]).

The version of the Jensen integral inequality is given below:

Lemma 2.2. Let $\mathrm{f}$ be an integrable function defined on $(a ; b)$ and let $\varphi:(a ; b) \rightarrow \mathbb{R}$ be a convex function. If $\varphi$ of $\in L_{(a ; b)}$, then

$$
\phi\left(\frac{1}{b-a} \int_{a}^{b} f(t) d t\right) \leq \frac{1}{b-a}\left(\int_{a}^{b} \phi(f(t)) d t\right) .
$$

The above inequality is inverted if $\varphi$ is a concave function. The inequality in the Lemma 2.2 can be rewritten in the following forms.

- If $\phi$ is a convex function, then

$$
\left(\int_{a}^{b} \phi(f(t)) d t\right) \geq(b-a) \phi\left(\frac{1}{b-a} \int_{a}^{b} f(t) d t\right) .
$$

- If $\phi$ is a concave function, then 


$$
\left(\int_{a}^{b} \phi(f(t)) d t\right) \leq(b-a) \phi\left(\frac{1}{b-a} \int_{a}^{b} f(t) d t\right) .
$$

\section{MAIN RESULTS}

Theorem 3.1. Let $\mathrm{f}, \mathrm{g}$ be integrable positives functions on $[\mathrm{a} ; \mathrm{b}], \mu$ be a weight function on $(\mathrm{a} ; \mathrm{b})$ and

$$
F_{\mu}(x)=\int_{0}^{x} f(t) \mu(t) d t
$$

If $\mathrm{g}$ is non-decreasing and $\mu$ is non-increasing, then

(i) for $1 \leq p \leq q$,

$$
\begin{gathered}
q^{\frac{p}{q}} \int_{a}^{b} \frac{\left(\mathrm{F}_{\mu}\right)^{p}(x)}{g(x)} d x \\
\leq \mu^{p}(a)(b-a)^{1-\frac{p}{q}}\left[(b-a)^{q} \int_{a}^{b} \frac{f^{q}(x)}{g^{\frac{q}{p}}(x)} d x-\int_{a}^{b}(x-a)^{q} \frac{f^{q}(x)}{g^{\frac{q}{p}}(x)} d x\right]^{\frac{p}{q}},
\end{gathered}
$$

(ii) for $0<q \leq p<1$,

$$
\begin{gathered}
q^{\frac{p}{q}} \int_{a}^{b} \frac{\left(\mathrm{F}_{\mu}\right)^{p}(x)}{g(x)} d x \\
\geq \frac{\mu^{p}(b)}{g(b)}(b-a)^{1-\frac{p}{q}}\left[(b-a)^{q} \int_{a}^{b} f^{q}(x) d x-\int_{a}^{b}(x-a)^{q} f^{q}(x) d x\right]^{\frac{p}{q}} .(7)
\end{gathered}
$$

Proof. ( $i$ ) For $1 \leq p \leq q$, Apply the Hölder inequality for $\frac{1}{p}+\frac{1}{p^{\prime}}=1$, we get

$$
\begin{gathered}
\int_{a}^{b} \frac{\left(\mathrm{F}_{\mu}\right)^{p}(x)}{g(x)} d x=\int_{a}^{b} g^{-1}(x)\left(\int_{0}^{x} f(t) \mu(t) d t\right)^{p} d x \\
\leq \int_{a}^{b} g^{-1}(x)\left\{\left(\int_{0}^{x} f^{p}(t) \mu(t) d t\right)^{\frac{1}{p}}\left(\int_{0}^{x} \mu(t) d t\right)^{\frac{1}{\boldsymbol{p}^{\prime}}}\right\}^{p} d x \\
=\int_{a}^{b} g^{-1}(x)\left(\int_{0}^{x} f^{p}(t) \mu(t) d t\right)\left(\int_{0}^{x} \mu(t) d t\right)^{p-1} d x
\end{gathered}
$$

Using the inequality (3) and since $\mu$ is non-increasing function, we deduce that

$$
\int_{a}^{b} \frac{\left(\mathrm{F}_{\mu}\right)^{p}(x)}{g(x)} d x \leq \int_{a}^{b} g^{-1}(x)\left(\int_{0}^{x} \mu(t) d t\right)^{\boldsymbol{p}-\frac{\boldsymbol{P}}{\boldsymbol{q}}}\left(\int_{0}^{x} f^{q}(t) \mu(t) d t\right)^{\frac{\boldsymbol{p}}{\boldsymbol{q}}} d x
$$




$$
\begin{gathered}
\leq \int_{a}^{b} g^{-1}(x) \mu^{p}(a)(\boldsymbol{x}-\boldsymbol{a})^{(\boldsymbol{q}-1)-\frac{\boldsymbol{p}}{\boldsymbol{q}}}\left(\int_{0}^{\boldsymbol{x}} f^{q}(t) d t\right)^{\frac{\boldsymbol{p}}{\boldsymbol{q}}} d x \\
=\mu^{\mathrm{p}}(\mathrm{a}) \int_{\mathrm{a}}^{\mathrm{b}}(\mathrm{H}(\mathrm{x}))^{\frac{\mathrm{p}}{\mathrm{q}}} \mathrm{dx}
\end{gathered}
$$

Where

$$
H(x)=\int_{0}^{x} g^{-\frac{q}{p}}(x)(\boldsymbol{x}-\boldsymbol{a})^{(\boldsymbol{q}-1)} f^{q}(t) d t
$$

Let $\phi(x)=x^{\frac{p}{q}}$ be a concave function and $g$ be non-decreasing function, apply the inequality (5), hence

$$
\begin{aligned}
\int_{\mathrm{a}}^{\mathrm{b}}(\mathrm{H}(\mathrm{x}))^{\frac{\mathrm{p}}{\mathrm{q}}} \mathrm{dx} & =\int_{\mathrm{a}}^{\mathrm{b}} \phi(\mathrm{H}(\mathrm{x})) \mathrm{dx} \\
& \leq(\mathrm{b}-\mathrm{a}) \phi\left(\frac{1}{\mathrm{~b}-\mathrm{a}} \int_{\mathrm{a}}^{\mathrm{b}} \mathrm{H}(\mathrm{x}) \mathrm{dx}\right) \\
& =(b-a)^{1-\frac{p}{q}}\left(\int_{a}^{b} \int_{0}^{x} g^{-\frac{q}{p}}(x)(\boldsymbol{x}-\boldsymbol{a})^{(\boldsymbol{q}-\mathbf{1})} f^{q}(t) d t d x\right)^{\frac{p}{q}} \\
& =(b-a)^{1-\frac{p}{q}}\left(\int_{a}^{b} f^{q}(t) \int_{\boldsymbol{t}}^{\boldsymbol{b}} g^{-\frac{q}{p}}(x)(\boldsymbol{x}-\boldsymbol{a})^{(\boldsymbol{q}-1)} d x d t\right)^{\frac{p}{q}} \\
& \leq(b-a)^{1-\frac{p}{q}}\left(\int_{a}^{b} f^{q}(t) g^{-\frac{q}{p}}(t) \int_{\boldsymbol{t}}^{\boldsymbol{b}}(\boldsymbol{x}-\boldsymbol{a})^{(\boldsymbol{q}-\mathbf{1})} d x d t\right)^{\frac{p}{q}}
\end{aligned}
$$

Consequently

$$
\begin{aligned}
\int_{a}^{b} \frac{\left(\mathrm{F}_{\mu}\right)^{p}(x)}{g(x)} & d x \leq \mu^{p}(a)(b-a)^{1-\frac{p}{q}}\left(\frac{1}{q} \int_{a}^{b} f^{q}(t) g^{-\frac{q}{p}}(t)\left[(\boldsymbol{b}-\boldsymbol{a})^{\boldsymbol{q}}-(\boldsymbol{t}-\boldsymbol{a})^{\boldsymbol{q}}\right] d t\right)^{\frac{p}{q}} \\
& =\left(\frac{\mu(a)}{q^{\frac{1}{q}}}\right)^{p}(b-a)^{1-\frac{p}{q}}\left\{(\boldsymbol{b}-\boldsymbol{a})^{\boldsymbol{q}} \int_{a}^{b} \frac{f^{q}(t)}{g^{\frac{q}{p}}(t)} d t-\int_{a}^{b}(\boldsymbol{t}-\boldsymbol{a})^{\boldsymbol{q}} \frac{f^{q}(t)}{g^{\frac{q}{p}}(t)} d t\right\}^{\frac{p}{q}},
\end{aligned}
$$

thus we get (6) .

(ii) For $0<q \leq p<1$, using the reverse Hölder inequality, we get

$$
\int_{a}^{b} \frac{\left(\mathrm{F}_{\mu}\right)^{p}(x)}{g(x)} d x \geq \int_{a}^{b} g^{-1}(x)\left(\int_{0}^{x} f^{p}(t) \mu(t) d t\right)\left(\int_{0}^{x} \mu(t) d t\right)^{p-1} d x
$$

Apply the reverse of the inequality (3) and since $\mu$ is non-increasing function, we deduce that 


$$
\begin{aligned}
\int_{a}^{b} \frac{\left(\mathrm{F}_{\mu}\right)^{p}(x)}{g(x)} d x & \geq \int_{a}^{b} g^{-1}(x)\left(\int_{\mathbf{0}}^{\boldsymbol{x}} \mu(t) d t\right)^{\boldsymbol{p}-\frac{\boldsymbol{P}}{\boldsymbol{q}}}\left(\int_{\mathbf{0}}^{\boldsymbol{x}} f^{q}(t) \mu(t) d t\right)^{\frac{\boldsymbol{p}}{\boldsymbol{q}}} d x \\
& \geq \int_{a}^{b} g^{-1}(x) \mu^{p}(b)(x-a)^{(\boldsymbol{q}-\mathbf{1})-\frac{\boldsymbol{P}}{\boldsymbol{q}}}\left(\int_{\mathbf{0}}^{\boldsymbol{x}} f^{q}(t) d t\right)^{\frac{\boldsymbol{p}}{\boldsymbol{q}}} d x \\
& =\mu^{p}(b) \int_{a}^{b}(H(x))^{\frac{\boldsymbol{p}}{\boldsymbol{q}}} d x
\end{aligned}
$$

Let $\phi(x)=x^{\frac{p}{q}}$ be a convex function and $g$ be non-decreasing function, apply the inequality (4), hence

$$
\begin{aligned}
\int_{a}^{b}(H(x))^{\frac{p}{q}} d x & \geq(b-a)^{1-\frac{p}{q}}\left(\int_{a}^{b} \int_{0}^{x} g^{-\frac{q}{p}}(x)(\boldsymbol{x}-\boldsymbol{a})^{(\boldsymbol{q}-\mathbf{1})} f^{q}(t) d t d x\right)^{\frac{p}{q}} \\
& \leq(b-a)^{1-\frac{p}{q}}\left(\int_{a}^{b} f^{q}(t) g^{-\frac{q}{p}}(b) \int_{\boldsymbol{t}}^{\boldsymbol{b}}(\boldsymbol{x}-\boldsymbol{a})^{(\boldsymbol{q}-\mathbf{1})} d x d t\right)^{\frac{p}{q}} \\
& =\frac{(b-a)^{1-\frac{p}{q}}}{g(b)}\left(\int_{a}^{b} f^{q}(t) \int_{\boldsymbol{t}}^{\boldsymbol{b}}(\boldsymbol{x}-\boldsymbol{a})^{(\boldsymbol{q}-\mathbf{1})} d x d t\right)^{\frac{p}{q}}
\end{aligned}
$$

therefore

$$
\begin{aligned}
\int_{a}^{b} \frac{\left(\mathrm{F}_{\mu}\right)^{p}(x)}{g(x)} d x & \leq \frac{\mu^{p}(b)(b-a)^{1-\frac{p}{q}}}{g(b)}\left(\frac{1}{q} \int_{a}^{b} f^{q}(t)\left[(\boldsymbol{b}-\boldsymbol{a})^{q}-(\boldsymbol{t}-\boldsymbol{a})^{q}\right] d t\right)^{\frac{p}{q}} \\
& =\frac{\mu^{\mathrm{p}}(\mathrm{b})(\mathrm{b}-\mathrm{a})^{1-\frac{\mathrm{p}}{\mathrm{q}}}}{\mathrm{q}^{\frac{\mathrm{p}}{\mathrm{q}} \mathrm{g}(\mathrm{b})}}\left\{(\mathrm{b}-\mathrm{a})^{\mathrm{q}} \int_{\mathrm{a}}^{\mathrm{b}} \mathrm{f}^{\mathrm{q}}(\mathrm{t}) \mathrm{dt}-\int_{\mathrm{a}}^{\mathrm{b}} \mathrm{f}^{\mathrm{q}}(\mathrm{t})(\mathrm{t}-\mathrm{a})^{\mathrm{q}} \mathrm{dt}\right\}^{\frac{\mathrm{p}}{\mathrm{q}}} .
\end{aligned}
$$

So, the proof of Theorem 3.1. is complete.

In the same data on the functions $f, g$ and $\mu$, with $F_{\mu}(x)=\int_{0}^{x} f(t) \mu(t) d t$ and by reasoning analogously to the proof of Theorem 3.1, we obtain the following remarks.

Remark 3.1. If $g$ and $\mu$ are non-decreasing functions, then

(i) for $1 \leq p \leq q$,

$$
\begin{gathered}
q^{\frac{p}{q}} \int_{a}^{b} \frac{\left(\mathrm{F}_{\mu}\right)^{p}(x)}{g(x)} d x \\
\leq \mu^{p}(b)(b-a)^{1-\frac{p}{q}}\left[(b-a)^{q} \int_{a}^{b} \frac{f^{q}(x)}{g^{\frac{q}{p}}(x)} d x-\int_{a}^{b}(x-a)^{q} \frac{f^{q}(x)}{g^{\frac{q}{p}}(x)} d x\right]^{\frac{p}{q}},
\end{gathered}
$$

(ii) for $0<q \leq p<1$, 


$$
\begin{gathered}
q^{\frac{p}{q}} \int_{a}^{b} \frac{\left(\mathrm{F}_{\mu}\right)^{p}(x)}{g(x)} d x \\
\geq \frac{\mu^{p}(a)}{g(b)}(b-a)^{1-\frac{p}{q}}\left[(b-a)^{q} \int_{a}^{b} f^{q}(x) d x-\int_{a}^{b}(x-a)^{q} f^{q}(x) d x\right]^{\frac{p}{q}} .
\end{gathered}
$$

Remark 3.2. If $g$ is non-increasing and $\mu$ is non-decreasing, then

(i) for $1 \leq p \leq q$,

$$
\begin{gathered}
q^{\frac{p}{q}} \int_{a}^{b} \frac{\left(\mathrm{F}_{\mu}\right)^{p}(x)}{g(x)} d x \\
\leq \frac{\mu^{p}(b)}{g(b)}(b-a)^{1-\frac{p}{q}}\left[(b-a)^{q} \int_{a}^{b} f^{q}(x) d x-\int_{a}^{b}(x-a)^{q} f^{q}(x) d x\right]^{\frac{p}{q}},
\end{gathered}
$$

(ii) for $0<q \leq p<1$,

$$
\begin{gathered}
q^{\frac{p}{q}} \int_{a}^{b} \frac{\left(\mathrm{F}_{\mu}\right)^{p}(x)}{g(x)} d x \\
\geq \mu^{p}(a)(b-a)^{1-\frac{p}{q}}\left[(b-a)^{q} \int_{a}^{b} \frac{f^{q}(x)}{g^{\frac{q}{p}}(x)} d x-\int_{a}^{b}(x-a)^{q} \frac{f^{q}(x)}{g^{\frac{q}{p}}(x)} d x\right]^{\frac{p}{q}} .
\end{gathered}
$$

Remark 3.3. If $g$ and $\mu$ are non-increasing functions, then

(i) for $1 \leq \mathrm{p} \leq \mathrm{q}$,

$$
\begin{gathered}
q^{\frac{p}{q}} \int_{a}^{b} \frac{\left(\mathrm{F}_{\mu}\right)^{p}(x)}{g(x)} d x \\
\leq \frac{\mu^{p}(a)}{g(b)}(b-a)^{1-\frac{p}{q}}\left[(b-a)^{q} \int_{a}^{b} f^{q}(x) d x-\int_{a}^{b}(x-a)^{q} f^{q}(x) d x\right]^{\frac{p}{q}},
\end{gathered}
$$

(ii) for $0<q \leq p<1$,

$$
\begin{gathered}
q^{\frac{p}{q}} \int_{a}^{b} \frac{\left(\mathrm{F}_{\mu}\right)^{p}(x)}{g(x)} d x \\
\geq \mu^{p}(b)(b-a)^{1-\frac{p}{q}}\left[(b-a)^{q} \int_{a}^{b} \frac{f^{q}(x)}{g^{\frac{q}{p}}(x)} d x-\int_{a}^{b}(x-a)^{q} \frac{f^{q}(x)}{g^{\frac{q}{p}}(x)} d x\right]^{\frac{p}{q}} .
\end{gathered}
$$




\section{APPLICATIONS}

We now give some new consequences of the above results.

\subsection{The reverses weighted Hardy's type inequalities}

If we set $q=p$ in Theorem3.1., we obtain the following corollary.

Corollary 4.1. Let $f$, $g$ be integrable positives functions on $[a ; b], \mu$ be a weight function on (a; b) and

$$
\mathrm{F}_{\mu}(x)=\int_{0}^{x} f(t) \mu(t) d t
$$

If $\mathrm{g}$ is non-decreasing and $\mu$ is non-increasing then

(i) for $1 \leq p$,

$$
p \int_{a}^{b} \frac{\left(\mathrm{F}_{\mu}\right)^{p}(x)}{g(x)} d x \leq \mu^{p}(a)\left((b-a)^{p} \int_{a}^{b} \frac{f^{p}(x)}{g(x)} d x-\int_{a}^{b}(x-a)^{p} \frac{f^{q}(x)}{g(x)} d x\right),
$$

(ii) for $0<p<1$,

$$
p \int_{a}^{b} \frac{\left(\mathrm{F}_{\mu}\right)^{p}(x)}{g(x)} d x \geq \frac{\mu^{p}(b)}{g(b)}\left((b-a)^{p} \int_{a}^{b} f^{p}(x) d x-\int_{a}^{b}(x-a)^{p} f^{p}(x) d x\right) .
$$

According to remarks 3.1,3.2,3.3 above and with the same condition $q=p$, one can deduce new results, in a similar way to the inequalities (14) and (15) via to the monotonicity of the functions $g$ and $\mu$.

\subsection{The reverses Hardy's type inequalities}

If we set $\mu \equiv 1$ in Theorem3.1. and remarks 3.1,3.2,3.3, we result some new inequalities with two parameters in the following corollaries.

Corollary 4.2. Let $f, g$ be positive functions defined on $[a ; b]$ and $F(x)=\int_{a}^{x} f(t) d t$.

If $\mathrm{g}$ is non-decreasing, then

(i) for $1 \leq p \leq q$,

$$
q^{\frac{p}{q}} \int_{a}^{b} \frac{\mathrm{F}^{p}(x)}{g(x)} d x \leq(b-a)^{1-\frac{p}{q}}\left[(b-a)^{q} \int_{a}^{b} \frac{f^{q}(x)}{g^{\frac{q}{p}}(x)} d x-\int_{a}^{b}(x-a)^{q} \frac{f^{q}(x)}{g^{\frac{q}{p}}(x)} d x\right]^{\frac{p}{q}}
$$

(ii) for $0<q \leq p<1$,

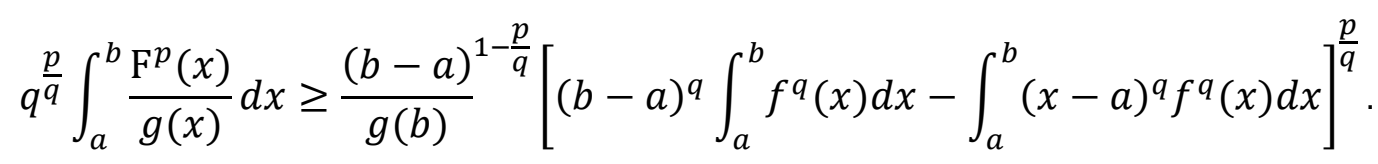

Inequalities (16) and (17) are new generalizations of inequalities (1) and (2).

Corollary 4.3. Let $f$, $g$ be positive functions defined on $[a ; b]$ and $F(x)=\int_{a}^{x} f(t) d t$. If $g$ is non-increasing, then 
(i) for $1 \leq p \leq q$,

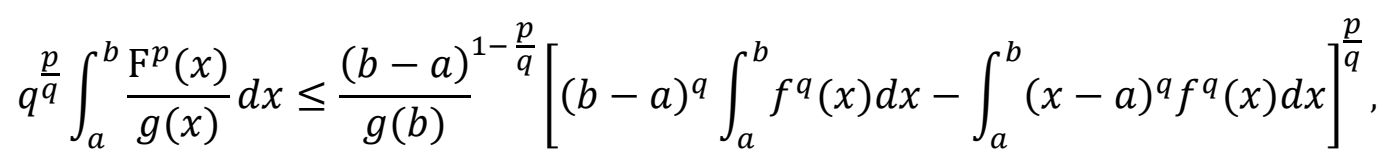

(ii) for $0<q \leq p<1$,

$$
q^{\frac{p}{q}} \int_{a}^{b} \frac{\mathrm{F}^{p}(x)}{g(x)} d x \geq(b-a)^{1-\frac{p}{q}}\left[(b-a)^{q} \int_{a}^{b} \frac{f^{q}(x)}{g^{\frac{q}{p}}(x)} d x-\int_{a}^{b}(x-a)^{q} \frac{f^{q}(x)}{g^{\frac{q}{p}}(x)} d x\right]^{\frac{p}{q}}
$$

The inequalities (18) and (19) are new results with two parameters in the case where $g$ is a non-increasing function. If we $\mathrm{p}=\mathrm{q}$ put in the Corollary4.2. we obtain the following new result.

Remark4.1 Let $f, g$ be positive functions defined on $[a ; b]$ and $F(x)=\int_{a}^{x} f(t) d t$. If $g$ is nonincreasing, then for $\mathrm{p} \geq 1$,

$$
p \int_{a}^{b} \frac{\mathrm{F}^{p}(x)}{g(x)} d x \leq \frac{1}{g(b)}\left((b-a)^{p} \int_{a}^{b} f^{p}(x) d x-\int_{a}^{b}(x-a)^{p} f^{p}(x) d x\right),
$$

for $0<p<1$,

$$
p \int_{a}^{b} \frac{F(x)^{p}}{g(x)} d x \geq(b-a)^{p} \int_{a}^{b} \frac{f^{p}(x)}{g(x)} d x-\int_{a}^{b}(x-a)^{p} \frac{f^{p}(x)}{g(x)} d x .
$$

\section{CONCLUSIONS}

By applying Hölder's inequality to two integrability parameters and Jensen's integral inequality, new generalization integral inequalities relating to the inverse-weighted Hardy inequalities have been established and proven. Some particular cases are studied according to the monotony of the functions.

Acknowledgements: This research is supported by DGRSDT. Algeria.

\section{REFERENCES}

[1] B. Benaissa, M.Z. Sarikaya, A. Senouci “On some new Hardy-type inequalities”, Math. Meth. Appl .Sci., 43, 8488-8495 (2020). https://doi.org/10.1002/mma.6503

[2] A. Khameli, Z. Dahmani, K. Freha, and M. Z .Sarikaya, "New Riemann-Liouville generalizations for some inequalities of Hardy type", Malaya J. Mat., 4(2), 277-283 (2016).

[3] B. Benaissa, M.Z. Sarikaya, "Some Hardy-type integral inequalities involving functions of two independent variables". Positivity, 25, 853-866 (2021). https://doi.org/10.1007/s11117$\underline{020-00791-5}$

[4] B.G Pachpatte, "On Hardy type integral inequalities for a functions of two variables", Demonstratio Math., XXVIII(2), (1995). https://doi.org/10.1515/dema-1995-0202

[5] B. Benaissa, A. Senouci "Some new integral inequalities through the Steklov operator", Math. Montis., 49, 49 - 56 (2020). https://doi:10.20948/mathmontis-2020-49-4

[6] B. Benaissa, "Some Inequalities on Time Scales Similar to Reverse Hardy's Inequality", Rad Hrvat. Akad. Znan. Umjet. Mat. Znan., (2021), Forthcoming papers (with pdf preview). 
[7] B. Benaissa, "More on Minkowski and Hardy integral inequality on time scales", Ricerche mat (2021). https://doi.org/10.1007/s11587-021-00595-Z

[8] B. Benaissa, "More on reverses of Minkowski's inequalities and Hardy's integral inequalities", Asian-Eur. J. Math., 13 (3) (2020). https://doi:10.1142/S1793557120500643

[9] W.T. Sulaiman, "Reverses of Minkowski's, Hölder's, and Hardy's integral inequalities", Int. J. Mod. Math. Sci., 1 (1), 14-24 (2012).

[10] Banyat Sroysang, "A Generalization of Some Integral Inequalities Similar to Hardy's Inequality", Math. AEterna, 3 (7), 593-596 (2013).

[11] S.S. Dragomir, A.K. Muhammad, A. Addisalem, "Refinement of the Jensen integral inequality", Open. Math., 14 (1), 221-228 (2016). https://doi:10.1515/math-2016-0020

Received December 5, 2021 